\title{
Sodium channel variants linked to symptom phenotype in SFN
}

Mutations in the sodium channel $\mathrm{Na}_{\mathrm{v}} 1.7$ that cause small fibre neuropathy (SFN) have variable functional effects on neurons, which might explain the variability of symptoms among patients with these disorders, according to a recent paper in Brain. "While we previously understood the ways in which $\mathrm{Na}_{\mathrm{v}} 1.7$ mutations cause pain, we are now beginning to dissect phenotype-genotype relationships at a fine-grained level," says Stephen Waxman, lead researcher of the study.

In their previous investigations, Waxman and colleagues identifed mutations in $\mathrm{Na}_{\mathrm{v}} 1.7$ - a channel that is preferentially expressed in peripheral neurons-in $\sim 30 \%$ of their cohort with SFN. Most of these patients experienced severe autonomic dysfunction as well as pain; however, two patients, both of whom had an Arg185His mutation in $\mathrm{Na}_{\mathrm{v}} 1.7$, displayed only minor autonomic symptoms. "We decided to compare the detailed electrophysiology and biophysics of the Arg185His mutation with a comparator mutation, Ile739Val, found in patients with severe autonomic dysfunction," explains Waxman.

The researchers transfected rodentderived dorsal root ganglion (DRG) neurons and sympathetic ganglion

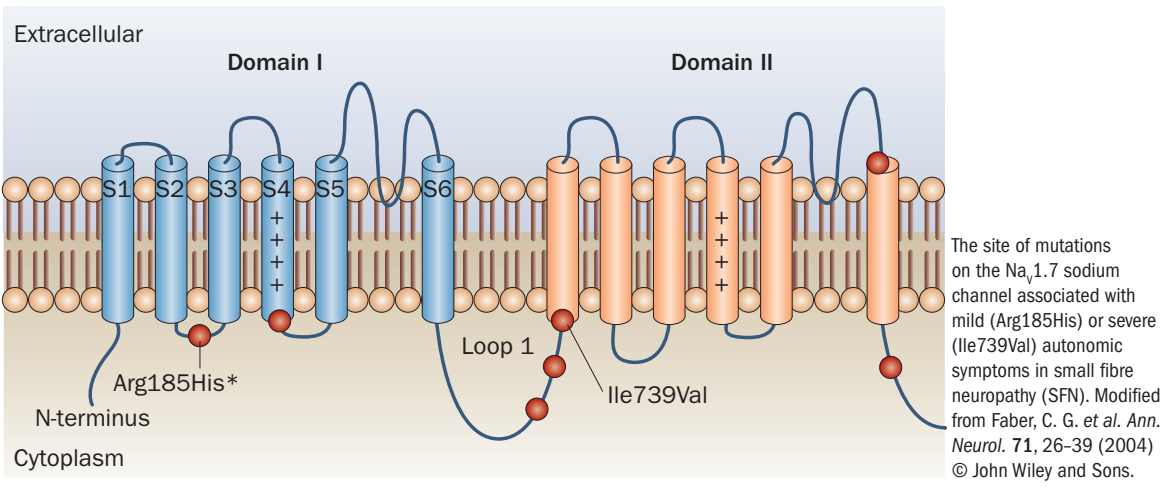

neurons with either of the two $\mathrm{Na}_{\mathrm{v}} 1.7$ mutants or wild-type $\mathrm{Na}_{\mathrm{v}} 1.7$, and performed functional analysis of the transfected cells using voltage-clamp and current-clamp electrophysiology. DRG neurons expressing the Arg185His mutant displayed increased excitability, but an unchanged resting potential. The DRG neurons transfected with the Ile739Val mutant were also hyperexcitable, but had depolarized resting potentials.

In sympathetic ganglion neurons, the Ile739Val mutation induced hypoexcitability. By contrast, the Arg185His mutation had no detectable effect on the excitability of these cells. "These findings explain why patients with the Ile739Val mutation experience autonomic dysfunction, while patients with the Arg185His mutation do not," states Waxman.

"Together with our observations on pharmacogenomic differences in patients carrying different $\mathrm{Na}_{\mathrm{v}} 1.7$ variants, and on gene polymorphisms that bias sensitivity to pain, we think it may be possible in the future to predict responsiveness to various pain medications," he concludes.

\section{Katy Malpass}

Original article Han, C. et al. Functional profiles of SCN9A
variants in dorsal root ganglion neurons and superior
cervical ganglion neurons correlate with autonomic
symptoms in small fibre neuropathy. Brain doi:10.1093/
brain/aws187

